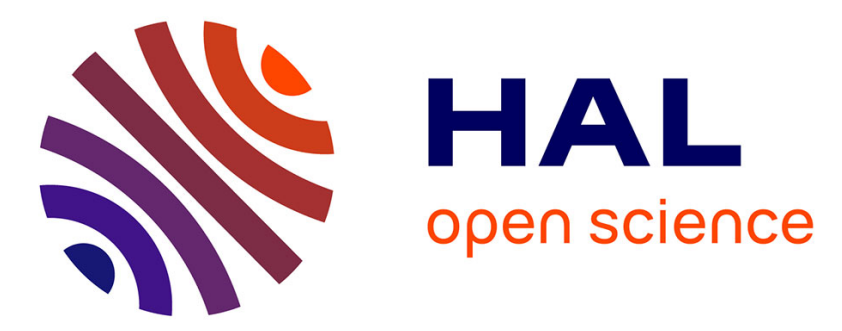

\title{
A Polynomial Algorithm for the Computation of Buffer Capacities with Throughput Constraint for Embedded System Design
}

Mohamed Benazouz, Olivier Marchetti, Alix Munier-Kordon, Pascal Urard

\section{- To cite this version:}

Mohamed Benazouz, Olivier Marchetti, Alix Munier-Kordon, Pascal Urard. A Polynomial Algorithm for the Computation of Buffer Capacities with Throughput Constraint for Embedded System Design. CIE IEEE International Conference on Computers \& Industrial Engineering, Jul 2009, Troyes, France. pp.690-695, 10.1109/ICCIE.2009.5223822 . hal-00368683

\section{HAL Id: hal-00368683 https://hal.science/hal-00368683}

Submitted on 17 Mar 2009

HAL is a multi-disciplinary open access archive for the deposit and dissemination of scientific research documents, whether they are published or not. The documents may come from teaching and research institutions in France or abroad, or from public or private research centers.
L'archive ouverte pluridisciplinaire HAL, est destinée au dépôt et à la diffusion de documents scientifiques de niveau recherche, publiés ou non, émanant des établissements d'enseignement et de recherche français ou étrangers, des laboratoires publics ou privés. 


\title{
A Polynomial Algorithm for the Computation of Buffer Capacities with Throughput Constraint for Embedded System Design
}

\author{
Mohamed Benazouz, Olivier Marchetti, Alix Munier-Kordon and Pascal Urard ${ }^{\dagger}$ \\ LIP6, Université Pierre et Marie Curie, Paris (France) \\ $\dagger$ Central R\&D - STMicroelectronics, Crolles (France) \\ Email: \{Mohamed.Benazouz, Olivier.Marchetti, Alix.Munier\}@lip6.fr, Pascal.Urard@st.com
}

\begin{abstract}
Marked Timed Weighted Event Graphs (in short MTWEG), which are a sub-class of Petri nets, are widely used for modelling practical industrial problems. In this paper, a central practical problem for the design of streaming (e.g. multimedia or network packet processing) applications is modelled using a MTWEG. The optimization problem tackled here consists then on finding an initial marking minimizing the overall number of tokens for a minimum given throughput.

If the firings of the transitions are periodic, this problem is NPcomplete and can be modelled using an Integer Linear Program. A general lower bound on the minimum overall capacity is then proved. If the initial MTWEG has a unique circuit, a polynomial time algorithm based on the resolution of a particular Diophantine equation is presented to solve it exactly. We lastly experiment it on an industrial example.
\end{abstract}

Index Terms-Timed Weighted Event Graphs, Periodic Schedule, Manufacturing System, Synchronous Dataflow, Buffer optimization.

\section{INTRODUCTION}

Cyclic scheduling problems, in which a set of generic tasks have to be performed infinitely often, have numerous practical applications in manufacturing systems or in the design of digital signal processing. Thus, many theoretical studies were devoted to these problems (for surveys, see [1], [2]).

Marked Weighted Timed Event Graphs (in short MTWEG) $\mathcal{G}$, which are a subclass of Petri Nets can be used to model some of these problems. Tasks corresponds to transitions with a fixed duration. Each place $p=\left(t_{i}, t_{j}\right)$ has exactly one input and one output transition: at the completion of a firing of $t_{i}$, $Z_{i}$ tokens are added to $p$. At the firing of $t_{j}, Z_{j}$ tokens are removed from $p$. If $Z_{i}=1$ for every transition $t_{i}, \mathcal{G}$ is a Marked Timed Event Graph (in short MTEG).

MTWEG and MTEG are widely used for modelling and solving practical cyclic scheduling problems. In the context of manufacturing systems, they are considered to model complex assembly lines. Workshop (resp. products) are usually modelled by transitions (resp. tokens). Between two successive transformations, products (i.e. tokens) have to be stored or

(C) 2009 IEEE. Personal use of this material is permitted. However, permission to reprint/republish this material for advertising or promotional purposes or for creating new collective works for resale or redistribution to servers or lists, or to reuse any copyrighted component of this work in other works must be obtained from the IEEE have to be moved from a workshop to another one. These amount of products, also called Work In Process (WIP in short), may have economical consequences. Therefore, the main problem for designers is to devise an initial configuration of WIP that allows the system to reach a given productivity and that uses the smallest amount of WIP.

MTWEG can also be considered for modelling data exchanges for streaming applications: transitions correspond to specific treatments. Places are associated with buffers. The total number of tokens of an initial marking is proportional to the overall surface of the memories. As the whole application has to be integrated on a single chip and satisfies high quality requirements, the surface minimization problem with throughput constraints is crucial for the design of these systems. However, designers of such systems usually model their system using Synchronous DataFlow Graph [3] (in short $\mathrm{SDF}$ ) which is an equivalent formalism.

For a given MTEG or MTWEG, the two fundamental questions are the existence of a schedule and the determination of the optimal throughput.

In the case of MTEG, these two problems are polynomially solved from a long time [4], [5], [6]. Thus, the minimization of a the sum of the initial markings for a minimum given throughput is in $N P$, and many authors developed efficient heuristics and exact methods to solve it (see. as example [7], [8], [9]). The NP-completeness of this last problem was proved recently in [10].

The existence of a polynomial algorithm for the liveness and the computation of the throughput of a MTWEG (or equivalently to a SDF) is a difficult question. Up to now, the time complexity of all the algorithms developed to answer these two fundamental questions is exponential in the worst case [11], [12]. The consequence is that the optimization problems on MTWEG are possibly not in $N P$ : the evaluation of the feasible solutions is not possible in polynomial time, which limits dramatically the existence of efficient algorithms. For example, Sauer [13] developed an algorithm to minimize the sum of the initial markings for a given throughput which evaluates a feasible solution using an exponential algorithm. The evaluation step of this algorithm limits significantly the size of the instances. In [14], [15], several buffer minimization problems with throughput constraint are modelled using an 
Integer Linear Program with an exponential number of equations. More recently, in [16], [17] authors have dealed with this problem with throughput constraint based on a state space exploration with model checking techniques.

Another way to circumvent this problem is to reduce the set of feasible solutions. Benabid et al. [18] developed a polynomial time algorithm for the computation of a periodic firing of the transitions. This result can be regarded as a generalization of Reiter's result for MTEG [19]. In the case of MTWEG, the existence of a periodic firing of the transitions is clearly more restrictive than the liveness. ùthe asap firing transition. The periodic scheduling policy is not necesseraly optimal for the throughput criteria. However, optimization problems, such as the minimization of the initial markings are now in $N P$ and efficient algorithms may be developed (even if the problem in $N P$-complete). As example, Wiggers et al. [20] developed a heuristic to solve it.

In this paper, we study the minimization of the overall number of initial tokens in a MTWEG for a periodic schedule with a given period. Section 2 is dedicated to basic definitions and the description of our problem. In Section 3, we show the modelling of a car radio using a MTWEG. Section 4 presents some important known basic results on periodic schedules. In Section 5, we show that our problem can be formulated using an Integer Linear Program and we show a first general lower bound on the overall places capacities. We prove in Section 6 and 7 that, if the MTWEG is a circuit, the determination of an optimal marking may be solved polynomially. In Section 8 , we apply our algorithm to the example presented in Section 3. We conclude in Section 9.

\section{MOdEL AND NOTATIONS}

\section{A. Basic definitions}

A Marked Timed Weighted Event Graph $\mathcal{G}=\left(T, P, l, M_{0}\right)$ is defined by a set of places $P=\left\{p_{1}, \ldots, p_{m}\right\}$ and a set of transitions $T=\left\{t_{1}, \ldots, t_{n}\right\}$. Every place $p \in P$ is defined between two transitions $t_{i}$ and $t_{j}$ and is denoted by $p=\left(t_{i}, t_{j}\right)$. For any transition $t_{i} \in T$, we set $\mathcal{P}^{+}\left(t_{i}\right)=\left\{p=\left(t_{i}, t_{j}\right) \in\right.$ $\left.P, t_{j} \in T\right\}$ and $\mathcal{P}^{-}\left(t_{i}\right)=\left\{p=\left(t_{j}, t_{i}\right) \in P, t_{j} \in T\right\}$. Moreover, it is supposed that $\mathcal{G}$ is strongly connected: for every couple of vertices $(x, y) \in(P \cup T)^{2}$, there exists a path in $\mathcal{G}$ from $x$ to $y$.

Every place $p \in P$ is initially marked by $M_{0}(p) \in \mathbb{N}$ tokens. We also suppose that every transition $t_{i}$ is valued by a strictly positive integer $Z_{i}$ and a processing time $\ell\left(t_{i}\right)$. If $t_{i}$ is fired at time $\tau, Z_{i}$ tokens are removed from every place $p \in \mathcal{P}^{-}\left(t_{i}\right)$. At time $\tau+\ell\left(t_{i}\right), Z_{i}$ tokens are added to every place $p \in$ $\mathcal{P}^{+}\left(t_{i}\right)$.

A place $p=\left(t_{i}, t_{j}\right)$ has a bounded capacity $F(p)>0$ if the number of tokens stored in $p$ can not exceed $F(p)$ : $\forall \tau \geq 0, M(\tau, p) \leq F(p)$. A MTWEG $\mathcal{G}=\left(T, P, M_{0}, l, F\right)$ is said to be a bounded capacity graph if the capacity of every place $p \in P$ is bounded by $F(p)$. It is proved in [21] that every place $p=\left(t_{i}, t_{j}\right)$ with bounded capacity may be replaced by a couple of places $\left(p_{1}=\left(t_{i}, t_{j}\right), p_{2}=\left(t_{j}, t_{i}\right)\right)$ denoted by $\left(p_{1}, p_{2}\right)_{c}$ with the initial marking $M_{0}\left(p_{1}\right)=M_{0}(p)$ and $M_{0}\left(p_{2}\right)=F(p)-M_{0}(p)$. So, in this paper, we only consider symmetric MTWEG: every place $p=\left(t_{i}, t_{j}\right)$ is associated with a backward place $p^{\prime}=\left(t_{j}, t_{i}\right)$ modelling the limited places capacity.

It is assumed that two successive firings of the same transition cannot overlap: this is modeled by a self-loop place $p=\left(t_{i}, t_{i}\right), \forall t_{i} \in T$ with $M_{0}(p)=Z_{i}$. For a sake of simplicity, these loops are not pictured.

The instantaneaous marking of a place $p \in P$ at time $\tau \geq 0$ is denoted by $M(\tau, p)$. Clearly, $M(0, p)=M_{0}(p)$.

For any couple of integers $(a, b) \in \mathbb{N}^{2}, \operatorname{gcd}(a, b)$ (resp. $\operatorname{lcm}(a, b)$ ) denotes the greatest common divisor (resp. least common multiple) of $a$ and $b$. For every couple of values $(p, q) \in \mathbb{N} \times \mathbb{N}^{\star}$, we set $\lceil p\rceil^{(q)}=\left\lceil\frac{p}{q}\right\rceil \cdot q$.

\section{EXAMPLE}

Let us consider a car-radio application described in [22]. The inputs of such systems are basically a MP3-reader and a cell phone. The output is a mixed sound from these two streams. Without any additional treatment, the output is reintroduced in the system through the cell phone, causing an echo effect. In order to obtain a pure speech in the cell phone, an additional input stream, corresponding to a microphone is added.

Figure 1 presents the streams and the main treatments. The first stream entrance, modelled by $t_{7}$ is the MP3 reader. $t_{10}$ corresponds to the entrance of the additional microphone. $t_{9}$ is the output. $t_{3}$ is the audio echo cancellation task. $t_{1}$ mixes the two input streams. $t_{5}$ produces a pure speech from the streams $t_{3}$ and the cell phone.

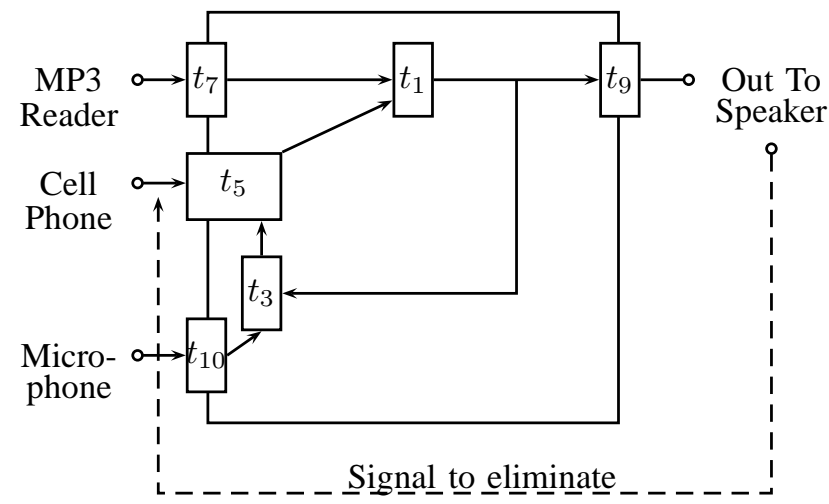

Fig. 1. Block diagram of a car-radio application

Figure 2 shows the modelling of the whole application by a MTWEG $\mathcal{G}$. Transitions $t_{2}, t_{4}, t_{6}$ and $t_{8}$ are simple rate convertors. Places model intermediate buffers of limited capacity between the components.

The processing times of the transitions are usually fixed by physical considerations and are presented by table I.

\section{PERIOdic SCHEDUleS}

\section{A. Schedules}

Let $\mathcal{G}$ be a MTWEG. A schedule is a function $s: T \times \mathbb{N}^{\star} \rightarrow$ $\mathbb{Q}^{+}$which associates, with any tuple $\left(t_{i}, q\right) \in T \times \mathbb{N}^{\star}$, the 
TABLE I

Processing TIMES $\ell\left(t_{i}\right), t_{i} \in T$ IN MILliseCONDS

\begin{tabular}{|c|c|c|c|c|c|c|c|c|c|c|}
\hline & $t_{1}$ & $t_{2}$ & $t_{3}$ & $t_{4}$ & $t_{5}$ & $t_{6}$ & $t_{7}$ & $t_{8}$ & $t_{9}$ & $t_{10}$ \\
\hline$l$ & $2.3 \times 10^{-2}$ & 10 & 9.091 & 0.125 & 0.125 & 10 & 24 & 10 & $2.3 \times 10^{-3}$ & 0.125 \\
\hline
\end{tabular}

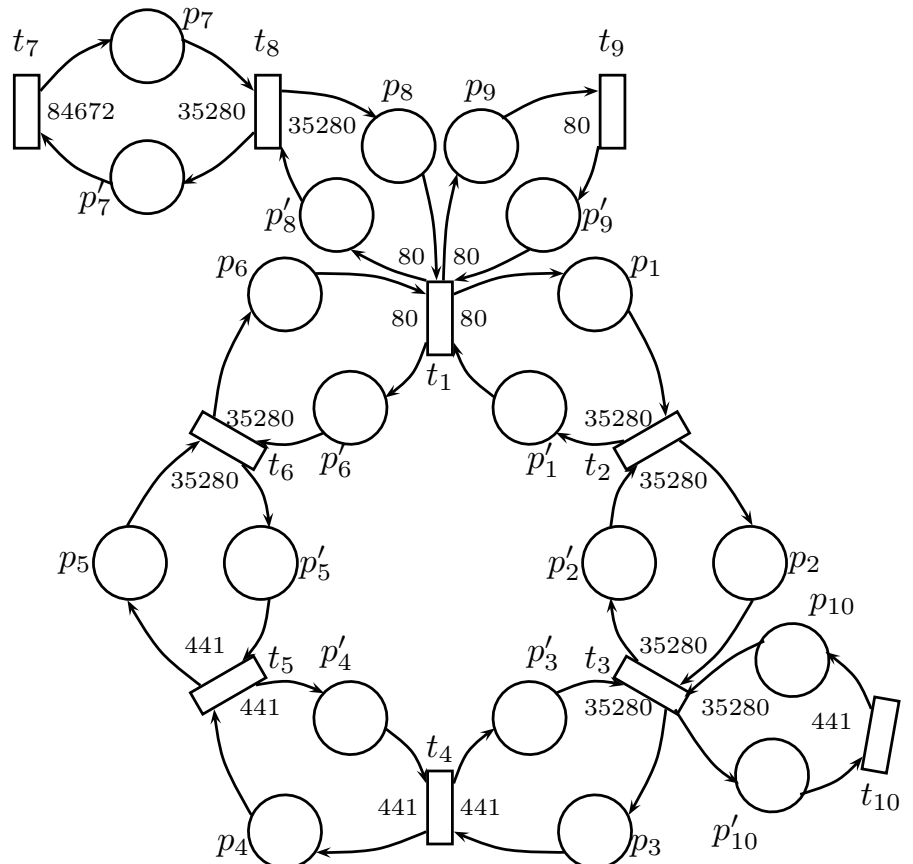

Fig. 2. A MTWEG $\mathcal{G}$ modelling a car-radio application

starting time of the $q$ th firing of $t_{i}$. There is a strong relationship between a schedule and the corresponding instantaneous marking. Indeed, a schedule is feasible if the number of tokens of every place $p=\left(t_{i}, t_{j}\right)$ remains non negative at each time instant.

It has been proved in [23] that the initial marking $M_{0}(p)$ of any place $p=\left(t_{i}, t_{j}\right)$ may be replaced by $\left\lfloor\frac{M_{0}(p)}{\operatorname{gcd}\left(Z_{i}, Z_{j}\right)}\right\rfloor \cdot \operatorname{gcd}\left(Z_{i}, Z_{j}\right)$ without any influence on $s$. Thus, we assume that the initial marking $M_{0}(p)$ of every place $p=\left(t_{i}, t_{j}\right) \in P$ is a multiple of $\operatorname{gcd}\left(Z_{i}, Z_{j}\right)$.

The throughput of a transition $t_{i}$ for a schedule $s$ is defined as

$$
\lambda^{s}\left(t_{i}\right)=\lim _{q \rightarrow \infty} \frac{q}{s\left(t_{i}, q\right)} .
$$

\section{B. Periodic schedules}

A schedule $s$ is periodic if there exists a vector $w=$ $\left(w_{1}, \ldots, w_{n}\right) \in \mathbb{Q}^{+n}$ such that, for any couple $\left(t_{i}, q\right) \in$ $T \times \mathbb{N}^{\star}, s\left(t_{i}, q\right)=s\left(t_{i}, 1\right)+(q-1) w_{i} . w_{i}$ is then the period of the transition $t_{i}$ and $\lambda^{s}\left(t_{i}\right)=\frac{1}{w_{i}}$ its throughput.

The following theorem proved in [18] characterizes the periodic schedule of a strongly connected MTWEG.

Theorem 1. For any feasible periodic schedule s, there exists $K \in \mathbb{Q}^{\star+}$ such that, for any couple of transitions $\left(t_{i}, t_{j}\right) \in T^{2}$, $\frac{w_{i}}{Z_{i}}=\frac{w_{j}}{Z_{j}}=K$. Moreover, $s$ is feasible iff, for any place $p=\left(t_{i}, t_{j}\right) \in P$,

$$
s\left(t_{j}, 1\right)-s\left(t_{i}, 1\right) \geq \ell\left(t_{i}\right)+K\left(Z_{j}-M_{0}(p)-g_{c d} d_{i, j},\right.
$$

where $\operatorname{gcd}_{i, j}=\operatorname{gcd}\left(Z_{i}, Z_{j}\right)$.

For our example, the throughput of the output must be equal to $44.1 \mathrm{kHz}$, thus $\frac{1}{w_{9}}=44.1 \mathrm{~ms}^{-1}$. Since $Z_{9}=80$, we get $K=\frac{w_{9}}{Z_{9}}=2.83 .10^{-4} \mathrm{~ms}$.

For any place $p=\left(t_{i}, t_{j}\right) \in P$, let us denote by $H(p)=$ $M_{0}(p)+g_{c} d_{i, j}-Z_{j}$ and $L(p)=\ell\left(t_{i}\right)$. For a circuit $c$, $H(c)=\sum_{p \in c} H(p)$ and $L(c)=\sum_{p \in c} L(p)$. Theorem 2 expresses a necessary and sufficient condition for the existence of a periodic schedule deduced from Bellman-Ford algorithm [24].

Theorem 2. There exists a periodic schedule iff, for every circuit $c$ of $G, H(c)>0$.

The minimum feasible value $K^{\text {opt }}$ of $K$ is then:

$$
K^{o p t}=\max _{c \in C(\mathcal{G})} \frac{L(c)}{H(c)}
$$

where $C(\mathcal{G})$ denotes the set of circuits of $\mathcal{G}$.

Numerous polynomial and pseudo-polynomial algorithms were developed to compute $K^{\text {opt }}$ (see. as example [25], [26]). An experimental study of these algorithms can be found in [27].

\section{General PROBLEM}

It is assumed here that $\mathcal{G}$ is a strongly connected MTWEG. The general problem is first presented and modelled by an Integer Linear Program. A lower bound of the overall capacity is then proved.

\section{A. Problem Formulation}

Let $\mathcal{G}=\left(T, P, l, M_{0}\right)$ be a symmetric MTWEG and $K \in$ $\mathbb{Q}^{+}$a fixed value for the period. The general problem tackled here is to find an initial marking $M_{0}(p), p \in P$ such that:

1) The overall capacity $\sum_{p \in P} F(p)=\sum_{p \in P} M_{0}(p)$ is minimum.

2) There exists a periodic schedule with a period at most equal to $\mathrm{K}$.

The problem may be formulated by the following Integer Linear Program $\Pi(K)$ :

$$
\begin{aligned}
& \min \left(\sum_{p \in P} M_{0}(p)\right) \\
& \begin{cases}\forall p=\left(t_{i}, t_{j}\right) \in P, & s\left(t_{j}, 1\right)-s\left(t_{i}, 1\right) \geq \ell\left(t_{i}\right)+ \\
& K \cdot\left(Z_{j}-M_{0}(p)-g c d_{i, j}\right) \\
\forall p=\left(t_{i}, t_{j}\right) \in P, & M_{0}(p)=k_{i j} \cdot g c d_{i, j} \\
\forall p=\left(t_{i}, t_{j}\right) \in P & k_{i j} \in \mathbb{N} \\
\forall t_{i} \in T, & s\left(t_{i}, 1\right) \geq 0\end{cases}
\end{aligned}
$$

The first inequality expresses the necessary and sufficient condition associated with a place $p$ on the first starting times of 
a feasible periodic schedule following Theorem 1 . The second equality comes from the restriction of $M_{0}(p), p=\left(t_{i}, t_{j}\right) \in P$ to multiples of $\operatorname{gcd}_{i, j}=\operatorname{gcd}\left(Z_{i}, Z_{j}\right)$.

\section{B. A general lower bound on the overall capacity}

Lemma 1. Let $\left(p, p^{\prime}\right)_{c}$ be a couple of place with $p=\left(t_{i}, t_{j}\right)$ and $p^{\prime}=\left(t_{j}, t_{i}\right)$. Let also the value

$$
F_{K}^{\star}\left(p, p^{\prime}\right)=\frac{l\left(t_{i}\right)+l\left(t_{j}\right)}{K}-2 g c d_{i j}+\left(Z_{i}+Z_{j}\right) .
$$

Then, for every feasible solution $M_{0}^{\star}$ of $\Pi(K), M_{0}^{\star}(p)+$ $M_{0}^{\star}\left(p^{\prime}\right) \geq\left\lceil F_{K}^{\star}\left(p, p^{\prime}\right)\right\rceil^{\left(\operatorname{gcd}_{i, j}\right)}$.

Proof: Let the circuit $c=\left(t_{i}, p, t_{j}, p^{\prime}, t_{i}\right)$. Then, $L(c)-$ $K H(c)=\ell\left(t_{i}\right)+\ell\left(t_{j}\right)-K\left(Z_{i}+Z_{j}-M_{0}^{\star}(p)-M_{0}^{\star}\left(p^{\prime}\right)-\right.$ $\left.2 \operatorname{gcd}_{i, j}\right)$. If $M_{0}^{\star}$ is feasible, we get $L(c)-K H(c) \leq 0$ and thus $M_{0}^{\star}(p)+M_{0}^{\star}\left(p^{\prime}\right) \geq \frac{\ell\left(t_{i}\right)+\ell\left(t_{j}\right)}{K}-2 \operatorname{gcd}_{i j}+\left(Z_{i}+Z_{j}\right)$. Since $M_{0}^{\star}(p)$ and $M_{0}^{\star}\left(p^{\prime}\right)$ are divisible by $\operatorname{gcd}_{i, j}$ we get the result.

For every couple of places $\left(p, p^{\prime}\right)_{c} \in P^{2},\left(p^{\prime}, p\right)_{c}$ is also a circuit. Theorem 3 is a simple outcome of Lemma 1 :

Theorem 3. $B=\sum_{\left(p, p^{\prime}\right)_{c} \in P^{2}, p=\left(t_{i}, t_{j}\right)} \frac{1}{2}\left\lceil F_{K}^{\star}\left(p, p^{\prime}\right)\right\rceil^{\left(\operatorname{gcd}_{i, j}\right)}$ is a lower bound on the overall capacity of a MTWEG $\mathcal{G}$ for a maximum fixed period $K \in \mathbb{Q}^{+}$.

\section{A polynomial Special Case}

Let us consider here that $\mathcal{G}$ is a double circuits of $n$ transitions defined as $c=\left(t_{1}, p_{1}, t_{2}, p_{2}, \ldots, t_{n}, p_{n}, t_{n+1}\right)$ with $t_{1}=t_{n+1}$ and $c^{\prime}=\left(t_{n+1}, p_{n}^{\prime}, t_{n}, \ldots, t_{2}, p_{1}^{\prime}, t_{1}\right)$. It is also assumed that $\operatorname{gcd}\left(Z_{1}, \ldots, Z_{n}\right)=1$. This assumption is not restrictive: if it is not true, it is proved in [23] that the integers $Z_{i}, t_{i} \in T$ can be replaced by $\frac{Z_{i}}{\operatorname{gcd}\left(Z_{1}, \ldots, Z_{n}\right)}$ without any influence on the existence and the period of a periodic schedule.

We first present a simplification of the ILP presented in the last section by eliminating the starting times of the first firings of the transitions. Then, we improve the lower bound presented previously. Lastly, we introduce a new system $\mathcal{S}$ and we show that, every solution of $\mathcal{S}$ gives an optimal solution. The resolution of $\mathcal{S}$ is detailed in the next section.

\section{A. Simplification of the Linear Program}

By Bellmann-Ford algorithm, there exists a solution $M_{0}(p)$, $p \in P$ for $\Pi(K)$ if every circuit $c$ verifies $L(c)-K H(c) \leq 0$. Thus, the system may be simplified by eliminating the starting times of the first firing of the transitions as follow:

1) For the first circuit $c$,

$$
\begin{aligned}
L(c)-K H(c)= & \sum_{i=1}^{n} \ell\left(t_{i}\right) \\
& +K \sum_{i=1}^{n}\left(Z_{i}-M_{0}\left(p_{i}\right)-\operatorname{gcd}_{i i+1}\right) \leq 0 .
\end{aligned}
$$

Thus,

$$
\sum_{i=1}^{n} M_{0}\left(p_{i}\right) \geq \frac{1}{2} \sum_{i=1}^{n} F_{K}^{\star}\left(p_{i}, p_{i}^{\prime}\right)
$$

and then, since the numbers of tokens are integer values,

$$
\sum_{i=1}^{n} M_{0}\left(p_{i}\right) \geq\left\lceil\frac{1}{2} \sum_{i=1}^{n} F_{K}^{\star}\left(p_{i}, p_{i}^{\prime}\right)\right\rceil .
$$

2) Similarly, we get for the circuit $c^{\prime}$,

$$
\sum_{i=1}^{n} M_{0}\left(p_{i}^{\prime}\right) \geq\left\lceil\frac{1}{2} \sum_{i=1}^{n} F_{K}^{\star}\left(p_{i}, p_{i}^{\prime}\right)\right\rceil .
$$

3) By Lemma 1 , circuits $\left(t_{i}, p_{i}, t_{i+1}, p_{i}^{\prime}, t_{i}\right), i \in\{1, \ldots, n\}$ induces

$$
M_{0}\left(p_{i}\right)+M_{0}\left(p_{i}^{\prime}\right) \geq\left\lceil F_{K}^{\star}\left(p_{i}, p\right)\right\rceil^{\left(\operatorname{gcd}_{i, i+1}\right)} .
$$

So, the system $\Sigma(K)$ to solve for a symmetric circuit is: $\min \left(\sum_{p \in P} M_{0}(p)\right)$

$\begin{cases} & \sum_{i=1}^{n} M_{0}\left(p_{i}\right) \geq\left\lceil\frac{1}{2} \sum_{i=1}^{n} F_{K}^{\star}\left(p_{i}, p_{i}^{\prime}\right)\right. \\ & \left.\sum_{i=1}^{n} M_{0}\left(p_{i}^{\prime}\right) \geq\left\lceil\frac{1}{2} \sum_{i=1}^{n} F_{K}^{\star}\left(p_{i}, p_{i}^{\prime}\right)\right\rceil\right] \\ \forall i \in\{1, \ldots, n\}, & M_{0}\left(p_{i}\right)+M_{0}\left(p_{i}^{\prime}\right) \geq\left\lceil F_{K}^{\star}\left(p_{i}, p_{i}^{\prime}\right)\right\rceil^{\left.\operatorname{gcd}_{i, i+1}\right)} \\ \forall p=\left(t_{i}, t_{j}\right) \in P, & M_{0}(p)=k_{i j} \cdot g c d_{i, j} \\ \forall p=\left(t_{i}, t_{j}\right) \in P, & k_{i j} \in \mathbb{N}\end{cases}$

\section{B. A Lower bound of the overall capacity}

From the two first equations, we get

$$
\sum_{i=1}^{n} M_{0}\left(p_{i}\right)+\sum_{i=1}^{n} M_{0}\left(p_{i}^{\prime}\right) \geq A,
$$

with $A=2 \times\left[\frac{1}{2} \sum_{i=1}^{n} F_{K}^{\star}\left(p_{i}, p_{i}^{\prime}\right)\right]$. So $A$ is a lower bound of the overall capacity. Moreover, $B=$ $\sum_{i=1}^{n}\left\lceil F_{K}^{\star}\left(p_{i}, p_{i}^{\prime}\right)\right\rceil^{\left(\operatorname{gcd}_{i, i+1}\right)}$ is also a lower bound by Theorem 3 .

So, $\max (A, B)$ is a lower bound of the overall capacity. However, this bound may be improved if $A>B$ :

Lemma 2. If $A>B$, then $A=B+1$.

Proof: Clearly,

$$
A=2 \times\left\lceil\frac{1}{2} \sum_{i=1}^{n} F_{K}^{\star}\left(p_{i}, p_{i}^{\prime}\right)\right\rceil \leq\left\lceil\sum_{i=1}^{n} F_{K}^{\star}\left(p_{i}, p_{i}^{\prime}\right)\right\rceil+1 .
$$

and

$$
\left\lceil\sum_{i=1}^{n} F_{K}^{\star}\left(p_{i}, p_{i}^{\prime}\right)\right\rceil \leq \sum_{i=1}^{n}\left\lceil F_{K}^{\star}\left(p_{i}, p_{i}^{\prime}\right)\right\rceil^{\left(\operatorname{gcd}_{i, i+1}\right)}=B .
$$

so, lemma holds.

Theorem 4. Let the index $j \in\{1, \ldots, n\}$ such that $\operatorname{gcd}_{j, j+1}$ is minimum. If $A>B$, then the minimal overall capacity for a period $K$ is equal to or greater than $B+\operatorname{gcd}_{j, j+1}$.

Proof: If the overall capacity of any couple of places $\left(p_{i}, p_{i}^{\prime}\right)$ is exactly $\left\lceil F_{K}^{\star}\left(p_{i}, p_{i}^{\prime}\right)\right]^{\left(\operatorname{gcd}_{i, i+1}\right)}$, then the overall capacity is $B$. If $A>B$, this solution is then not feasible. So, there is at least a couple $\left(p_{i}, p_{i}^{\prime}\right)_{c}$ with $i \in\{1, \ldots, n\}$ such that $M_{0}\left(p_{i}\right)+M_{0}\left(p_{i}^{\prime}\right)>\left\lceil F_{K}^{\star}\left(p_{i}, p_{i}^{\prime}\right)\right]^{\left(\operatorname{gcd}_{i, i+1}\right)}$. So a new lower bound of the capacity is $B+\operatorname{gcd}_{j, j+1}$. By Lemma 2, $B+\operatorname{gcd}_{j, j+1} \geq B+1=A$, and theorem follows. 


\section{Building another linear system}

The idea here is to build a simpler system $\mathcal{S}$ and to prove that an optimum solution for $\Sigma(K)$ can be deduced from every solution of $\mathcal{S}$.

Let us define the sequence $A_{i}, i \in\{1, \ldots, n\}$ as follows:

- If $B \geq A$, we set $A_{i}=\left\lceil F_{K}^{\star}\left(p_{i}, p_{i}^{\prime}\right)\right\rceil^{\left(\operatorname{gcd}_{i, i+1}\right)}$ for every $i \in\{1, \ldots, n\}$;

- Else, let $j \in\{1, \ldots, n\}$ such that $\operatorname{gcd}_{j, j+1}$ is minimum. We set $A_{j}=\left\lceil F_{K}^{\star}\left(p_{j}, p_{j}^{\prime}\right)\right\rceil^{\left(\operatorname{gcd}_{j, j+1}\right)}+\operatorname{gcd}_{j, j+1}$ and $A_{i}=$ $\left\lceil F_{K}^{\star}\left(p_{i}, p_{i}^{\prime}\right)\right\rceil^{\left(\operatorname{gcd}_{i, i+1}\right)}$ for every $i \in\{1, \ldots, n\}-\{j\}$.

Let

$$
Q=\sum_{i=1}^{n} A_{i}
$$

be the value of the overall capacity and $C=\left\lfloor\frac{Q}{2}\right\rfloor$. We also note, for every $i \in\{1, \ldots, n\}, a_{i}=\operatorname{gcd}_{i, i+1}$. It is proved in the next section that the following system $\mathcal{S}$ can be solved by a polynomial-time algorithm.

$$
\left\{\begin{array}{l}
C=\sum_{i=1}^{n} a_{i} x_{i} \\
\forall i \in\{1, \ldots, n\}, x_{i} \in \mathbb{N} \\
\forall i \in\{1, \ldots, n\}, 0 \leq a_{i} x_{i} \leq A_{i}
\end{array}\right.
$$

Theorem 5. Let $x_{i}^{\star}, i \in\{1, \ldots, n\}$ be a solution of $\mathcal{S}$. Then, the initial marking $M_{0}^{\star}$ defined as, $\forall i \in\{1, \ldots, n\}, M_{0}^{\star}\left(p_{i}\right)=$ $a_{i} x_{i}^{\star}$ and $M_{0}^{\star}\left(p_{i}^{\prime}\right)=A_{i}-a_{i} x_{i}^{\star}$ is an optimum solution of $\Sigma(K)$.

Proof: For every $i \in\{1, \ldots, n\}, M_{0}^{\star}\left(p_{i}\right)$ and $M_{0}^{\star}\left(p_{i}^{\prime}\right)$ are clearly divisible by $\operatorname{gcd}_{i, i+1}$. Moreover, $M_{0}^{\star}\left(p_{i}\right)+M_{0}^{\star}\left(p_{i}^{\prime}\right)=$ $A_{i} \geq\left\lceil F_{K}^{\star}\left(p, p_{i}^{\prime}\right)\right\rceil^{\left(\operatorname{gcd}_{i, i+1}\right)}$. Thus, the third inequality of $\Sigma(K)$ is fulfilled for every couple of places.

Two subcases must be considered:

1) If $B \geq A$, then $\frac{Q}{2}=\frac{B}{2} \geq \frac{A}{2}$. Thus, since $\frac{A}{2}$ is an integer value, $C=\left\lfloor\frac{Q}{2}\right\rfloor \geq \frac{A}{2}$. Now,

$$
\sum_{i=1}^{n} M_{0}^{\star}\left(p_{i}\right)=C \geq \frac{A}{2}=\left\lceil\frac{1}{2} \sum_{i=1}^{n} F_{K}^{\star}\left(p_{i}, p_{i}^{\prime}\right)\right\rceil
$$

and the first inequality of $\Sigma(K)$ is fullfilled.

On the same way,

$$
\sum_{i=1}^{n} M_{0}^{\star}\left(p_{i}^{\prime}\right)=\left\lceil\frac{Q}{2}\right\rceil \geq\left\lceil\frac{1}{2} \sum_{i=1}^{n} F_{K}^{\star}\left(p_{i}, p_{i}^{\prime}\right)\right\rceil
$$

and the second inequality of $\Sigma(K)$ is also verified. Lastly, the overall capacity is $Q=\sum_{i=1}^{n} A_{i}=B$, thus it is minimum.

2) Let us suppose now that $B<A$. Then, by Theorem 4 , $A=B+1$ and

$$
\frac{Q}{2}=\frac{B+a_{j}}{2}=\frac{a_{j}-1}{2}+\frac{A}{2} .
$$

Since $a_{j} \geq 1$ and $\frac{A}{2}$ is an integer,

$$
C=\left\lfloor\frac{Q}{2}\right\rfloor \geq\left\lceil\frac{1}{2} \sum_{i=1}^{n} F_{K}^{\star}\left(p_{i}, p_{i}^{\prime}\right)\right\rceil,
$$

Since $\sum_{i=1}^{n} M_{0}^{\star}\left(p_{i}\right)=C$ and $\sum_{i=1}^{n} M_{0}^{\star}\left(p_{i}^{\prime}\right)=\left\lceil\frac{Q}{2}\right\rceil$, the two first inequalities of $\Sigma(K)$ are verified.

Lastly, the overall capacity equals $Q=B+a_{i}$ and is minimum by Theorem 4 , which completes the proof.

\section{RESOLUTION OF $\mathcal{S}$}

In this section, a polynomial time-algorithm is developed to solve the system $\mathcal{S}$. We first present two technical lemmas expressing inequalities on $C$ and the sequence $A_{i}, i \in$ $\{1, \ldots, n\}$. Then, a three steps algorithm is detailed to solve $\mathcal{S}$.

\section{A. Technical properties}

The two following lemmas express important technical properties on $A_{i}, i \in\{1 \ldots, n\}$ and $C$ :

Lemma 3. $\forall i \in\{1, \ldots, n\}, A_{i}>Z_{i}+Z_{i+1}-2 a_{i}$.

Lemma 4. $\sum_{i=1}^{n}\left(Z_{i}-a_{i}\right) \leq C \leq \sum_{i=1}^{n} A_{i}-\sum_{i=1}^{n}\left(Z_{i}-a_{i}\right)$.

\section{B. Step 1 for solving $\mathcal{S}$}

The system $\sum_{i=1}^{n} a_{i} x_{i}=\operatorname{gcd}\left(a_{1}, \ldots, a_{n}\right)$ with $x_{i} \in \mathbb{Z}$ is a linear diophantine equation and can be solved by a generalization of the extended euclidean algorithm by a time-complexity algorithm bounded by $\mathcal{O}\left(n \log \left(\max \left(a_{1}, \ldots, a_{n}\right)^{2}\right)\right)$.

For our problem, we have $\operatorname{gcd}\left(a_{1}, \ldots, a_{n}\right)=$ $\operatorname{gcd}\left(Z_{1}, \ldots, Z_{n}\right)=1$. So, a solution to $C=\sum_{i=1}^{n} a_{i} x_{i}$ with $x_{i} \in \mathbb{Z}$ can be easily obtained. Let us denote it by $\hat{X}=\left(\hat{x}_{1}, \ldots, \hat{x}_{n}\right)$.

\section{Step 2 for solving $\mathcal{S}$}

The aim now is to build, from $\hat{X}$, another solution $\bar{X}$ to the equality $C=\sum_{i=1}^{n} a_{i} x_{i}$ with $X \in \mathbb{N}^{n}$. Let us build the sequence of integers $\Delta_{k}, k \in\{0, \ldots, n\}$ as follows:

1) $\Delta_{0}=0, \Delta_{n}=0$

2) for any $i \in\{1, \ldots, n-1\}, \Delta_{i}$ must be divisible by $\operatorname{lcm}\left(a_{i}, a_{i+1}\right)$ and the inequalities $0 \leq a_{i} \bar{x}_{i}-\Delta_{i-1}+$ $\Delta_{i}<Z_{i+1}$ must be true.

Observe that, since $Z_{i+1}$ is divisible by $\operatorname{lcm}\left(a_{i}, a_{i+1}\right)$, there are at least $\operatorname{lcm}\left(a_{i}, a_{i+1}\right)$ values in the integers interval $\left[0, Z_{i+1}\left[\right.\right.$ and the sequence $\Delta_{i}$ exits. Moreover, since $a_{i} \hat{x}_{i}, \Delta_{i}$ and $\Delta_{i-1}$ are all divisble by $a_{i}, a_{i} \hat{x}_{i}-\Delta_{i-1}+\Delta_{i} \leq Z_{i+1}-a_{i}$.

We set, for every $i \in\{1, \ldots, n-1\}, \bar{x}_{i}=\hat{x}_{i}-\frac{\bar{\Delta}_{i-1}}{a_{i}}+\frac{\Delta_{i}}{a_{i}}$.

Lemma 5. $\bar{X}=\left(\bar{x}_{1}, \ldots, \bar{x}_{n}\right) \in \mathbb{N}^{n}$ and verifies $C=$ $\sum_{i=1}^{n} a_{i} \bar{x}_{i}$.

Proof: By definition of $\bar{X}$,

$$
\sum_{i=1}^{n} a_{i} \bar{x}_{i}=\sum_{i=1}^{n} a_{i} \hat{x}_{i}-\sum_{i=1}^{n} \Delta_{i-1}+\sum_{i=1}^{n} \Delta_{i} .
$$

Now, since $\Delta_{0}=\Delta_{n}=0$,

$$
\sum_{i=1}^{n} a_{i} \bar{x}_{i}=\sum_{i=1}^{n} a_{i} \hat{x}_{i}-\sum_{i=1}^{n-1} \Delta_{i}+\sum_{i=1}^{n-1} \Delta_{i}
$$


and thus, $C=\sum_{i=1}^{n} a_{i} \bar{x}_{i}$.

Clearly, $\bar{X} \in \mathbb{Z}^{n}$. So, we must check that, for every $i \in$ $\{1, \ldots, n\}, \bar{x}_{i} \geq 0$.

1) This is true by definition of $\Delta_{i}$ for $i \in\{1, \ldots, n-1\}$.

2) Now, for every $i \in\{1, \ldots, n-1\}, a_{i} \bar{x}_{i} \leq Z_{i+1}-a_{i}$ and

$$
a_{n} \bar{x}_{n}=C-\sum_{i=1}^{n-1} a_{i} \bar{x}_{i} \geq C-\sum_{i=1}^{n-1}\left(Z_{i+1}-a_{i}\right) .
$$

By Lemma 4,

$$
C-\sum_{i=1}^{n-1}\left(Z_{i+1}-a_{i}\right) \geq Z_{1}-a_{n} \geq 0
$$

and thus $\bar{x}_{n} \geq 0$.

\section{Step 3 for solving $\mathcal{S}$}

We compute now from $\bar{X}$ a solution $X^{\star}$ for system $\mathcal{S}$. Let us build the sequence of positive integers $\Phi_{k}, k \in\{1, \ldots, n+1\}$ as follows:

1) $\Phi_{n+1}=0, \Phi_{1}=1$;

2) for any $i \in\{2, \ldots, n\}$, if $a_{i} \bar{x}_{i}+\Phi_{i+1}>A_{i}$, then compute $\Phi_{i}$ such that $A_{i}-Z_{i}<a_{i} \bar{x}_{i}+\Phi_{i+1}-\Phi_{i} \leq A_{i}$ and $\Phi_{i}$ is divisible by $\operatorname{lcm}\left(a_{i-1}, a_{i}\right)$. Otherwise, set $\Phi_{i}=0$.

As previously, the sequence $\Phi_{i}$ exists since $Z_{i}$ is divisible by $\operatorname{lcm}\left(a_{i-1}, a_{i}\right)$, so there are at least $\operatorname{lcm}\left(a_{i-1}, a_{i}\right)$ values in the integer interval $\left.] A_{i}-Z_{i}+1, A_{i}\right]$. Moreover, $A_{i}-Z_{i}$ and $a_{i} \bar{x}_{i}+\Phi_{i+1}-\Phi_{i}$ are divisible by $a_{i}$, so the first inequality becomes $A_{i}-Z_{i}-a_{i} \leq a_{i} \bar{x}_{i}+\Phi_{i+1}-\Phi_{i} \leq A_{i}$

We set, for every $i \in\{1, \ldots, n-1\}, x_{i}^{\star}=\bar{x}_{i}+\frac{\Phi_{i+1}}{a_{i}}-\frac{\Phi_{i}}{a_{i}}$. The proof of Theorem 6 is similar to Lemma 5 .

Theorem 6. $X^{\star}=\left(x_{1}^{\star}, \ldots, x_{n}^{\star}\right) \in \mathbb{N}^{n}$ is a solution to system $\mathcal{S}$.

\section{ApPlication to The CAR-RAdio}

Table II summarizes the values obtained for our example.

TABLE II

OPTIMAL INITIAL MARKINGS FOR THE MTWEG PICTURED BY FIGURE 2

\begin{tabular}{|l|c|c|c|c|c|}
\hline Buffers & $a_{i}$ & $F_{K}^{\star}$ & {$\left[F_{K}^{\star}\right]^{\left(a_{i}\right)}$} & $M_{0}^{\star}\left(p_{i}\right)$ & $M_{0}^{\star}\left(p_{i}^{\prime}\right)$ \\
\hline$\left(p_{1}, p_{1}^{\prime}\right)$ & 80 & 70560 & $882 a_{1}$ & $113 a_{1}$ & $769 a_{1}$ \\
\hline$\left(p_{2}, p_{2}^{\prime}\right)$ & 35280 & 67353,048 & $2 a_{2}$ & 0 & $2 a_{2}$ \\
\hline$\left(p_{3}, p_{3}^{\prime}\right)$ & 441 & 67353,048 & $153 a_{3}$ & $55 a_{3}$ & $98 a_{3}$ \\
\hline$\left(p_{4}, p_{4}^{\prime}\right)$ & 441 & 882 & $2 a_{4}$ & $2 a_{4}$ & 0 \\
\hline$\left(p_{5}, p_{5}^{\prime}\right)$ & 441 & 70560 & $160 a_{5}$ & $160 a_{5}$ & 0 \\
\hline$\left(p_{6}, p_{6}^{\prime}\right)$ & 80 & 70560 & $882 a_{6}$ & $882 a_{6}$ & 0 \\
\hline$\left(p_{7}, p_{7}^{\prime}\right)$ & 7056 & 225792 & $32 a_{7}$ & 0 & $32 a_{7}$ \\
\hline$\left(p_{8}, p_{8}^{\prime}\right)$ & 80 & 70560 & $882 a_{8}$ & 0 & $882 a_{8}$ \\
\hline$\left(p_{9}, p_{9}^{\prime}\right)$ & 80 & 160 & $2 a_{9}$ & 0 & $2 a_{9}$ \\
\hline$\left(p_{10}, p_{10}^{\prime}\right)$ & 441 & 67353,048 & $153 a_{10}$ & 0 & $153 a_{10}$ \\
\hline
\end{tabular}

The initial marking for the places from the circuit $c=$ $\left(t_{1}, p_{1}, t_{2}, \ldots, t_{6}, p_{6}, t_{1}\right)$ was computed using the algorithm developed here. We obtained for the lower bounds $B=$ 350595 and $A=347270$. Since $B>A, Q=B=350595$ and $C=\left\lfloor\frac{Q}{2}\right\rfloor=175297$. The vectors obtained for the three steps are $\hat{X}=(215 \cdot C, 0,-39 \cdot C, 0,0,0), \bar{X}=$ $(113,0,0,0,57,1764)$ and $X^{\star}=(113,0,55,2,160,882)$.

For any couple of places $\left(p_{i}, p_{i}^{\prime}\right) \in P$ which are not in $c$, the minimum capacity of the buffer is $\left\lceil F_{K}^{\star}\left(p_{i}, p_{i}^{\prime}\right)\right\rceil^{\left(a_{i}\right)}$. These buffers are initially empty, so $M_{0}^{\star}\left(p_{i}\right)=0$. If we set $M_{0}^{\star}\left(p_{i}^{\prime}\right)=$ $\left\lceil F_{K}^{\star}\left(p_{i}, p_{i}^{\prime}\right)\right\rceil^{\left(a_{i}\right)}$, we obtain a feasible solution for the system $\Sigma(K)$. Thus, it is an optimal initial marking.

If we compare our numerical results to [22], our results are slightly better. As example, for the couple $\left(p_{3}, p_{3}^{\prime}\right)$, they get a capacity of $158 a_{3}$, which is not minimum. Moreover, they supposed that a buffer is either initially full or empty, which limits solutions space and allows them to cut circuits. Lastly, time complexity of their algorithm is unknown.

\section{CONCLUSION}

We have developed in this paper a polynomial time algorithm for the minimization of the overall number of tokens for a minimum throughput. We proved that this problem can be modelled using an Integer Liner Program. A pertinent lower bound of the overall number of tokens is easily deduced from this formulation. We also proved then that if the initial MTWEG has a unique circuit, the problem considered is equivalent to a specific Linear Diophantine problem solvable by a polynomial time algorithm. This last algorithm was considered to solve exactly a practical application.

\section{REFERENCES}

[1] C. Hanen and A. Munier, "Cyclic scheduling on parallel processors: An overview," in Scheduling theory and its applications, P. Chrétienne, E. G. Coffman, J. K. Lenstra, and Z. Liu, Eds. J. Wiley and sons, 1994.

[2] M. Middendorf and V. G. Timkovsky, "On scheduling cycle shops: Classification, complexity and approximation," Journal of Scheduling, vol. 5, no. 2, pp. 135-169, 2002.

[3] E. A. Lee and D. G. Messerschmitt, "Synchronous data flow," IEEE Proceedings of the IEEE, vol. 75, no. 9, 1987.

[4] F. Commoner, A. W. Holt, S. Even, and A. Pnueli, "Marked directed graphs.” J. Comput. Syst. Sci., vol. 5, no. 5, pp. 511-523, 1971.

[5] P. Chrétienne, "Les réseaux de petri temporisés," Ph.D. dissertation, Thèse d'état, Université Pierre et Marie Curie, 1983.

[6] C. V. Ramamoorthy and G. S. Ho, "Performance evaluation of asynchronous concurrent systems using petri nets," IEEE Transactions on Software Engineering, vol. SE-6, no. 5, pp. 440-449, September 1980.

[7] S. Gaubert, "An algebraic method for optimizing resources in timed event graphs," in 9th conference on Analysis and Optimization of Systems, vol. 144. Springer, 1990.

[8] A. Giua, A. Piccaluga, and C. Seatzu, "Firing rate optimization of cyclic timed event graphs," Automatica, vol. 38, no. 1, 2002.

[9] S. Laftit, J.-M. Proth, and X. Xie, "Optimization of invariant criteria for event graphs," IEEE Transactions on Automatic Control, vol. 37, no. 5, 1989.

[10] O. Marchetti, "Dimensionnement des mémoires pour systèmes embarqués," Ph.D. dissertation, Université Pierre et Marie Curie, 2006.

[11] E. Teruel, P. Chrzastowski-Wachtel, J. M. Colom, and M. Silva, "On weighted T-systems," in Proocedings of the 13th Internationnal Conference on Application and Theory of Petri Nets 1992, Lecture Notes in Computer Science, vol. 616. Springer, 1992.

[12] A. Munier, "Régime asymptotique optimal d'un graphe d'événements temporisé généralisé: application à un problème d'assemblage," RAIROAutomatique Productique Informatique Industrielle, vol. 27, no. 5, pp. 487-513, 1993.

[13] N. Sauer, "Marking optimization of weighted marked graph," Discrete Event Dynamic Systems, vol. 13, no. 3, pp. 245-262, 2003. 
[14] R. Govindarajan and G. Gao, "Rate-optimal shcedule for multi-rate dsp computations," Journal of VLSI Signal Processing, no. 9, pp. 211-235, 1995.

[15] R. Govindarajan, G. R. Gao, and P. Desai, "Minimizing memory requirements in rate-optimal schedule in regular dataflow networks," Journal of VLSI Signal Processing, vol. 31, no. 3, 2002.

[16] S. Stuijk, M. Geilen, and T. Basten, "Exploring trade-offs in buffer requirements and throughput constraints for synchronous dataflow graphs," in 43rd Design Automation Conference, DAC 2006. ACM Press, 2006.

[17] A. H. Ghamarian, M. Geilen, T. Basten, and S. Stuijk, "Parametric throughput analysis of synchronous data flow graphs," in DATE, 2008, pp. 116-121.

[18] A. Benabid, C. Hanen, O. Marchetti, and A. M. Kordon, "Periodic schedules for unitary timed weighted event graphs," in Conference ROADEF'08. Clermont-Ferrand: Presses Universitaires de l'Universite Blaise Pascal, 2008, pp. 17-31.

[19] R. Reiter, "Scheduling parallel computations," Journal of the Association for Computing Machinery, vol. 15, no. 4, pp. 590-599, 1968.

[20] M. Wiggers, M. Bekooij, P. Jansen, and G. Smit, "Efficient computation of buffer capacities for multi-rate real-time systems with back-pressure," in CODES+ISSS '06: Proceedings of the 4th international conference on Hardware/software codesign and system synthesis. New York, NY, USA: ACM, 2006, pp. 10-15.

[21] O. Marchetti and A. Munier-Kordon, "Minimizing place capacities of weighted event graphs for enforcing liveness," Discrete Event Dynamic Systems, vol. 18, no. 1, pp. 91-109, 2008.

[22] M. H. Wiggers, M. J. G. Bekooij, P. G. Jansen, and G. J. M. Smit, "Efficient computation of buffer capacities for cyclo-static real-time systems with back-pressure," in RTAS '07: Proceedings of the 13th IEEE Real Time and Embedded Technology and Applications Symposium. Washington, DC, USA: IEEE Computer Society, 2007, pp. 281-292.

[23] O. Marchetti and A. Munier-Kordon, "A sufficient condition for the liveness of weighted event graphs," To appear in EJOR, LIP6 Research Report, http://ftp.lip6.fr/lip6/reports/2005/lip6-2005-004.pdf, 2008.

[24] T. H. Cormen, C. E. Leiseerson, R. Rivest, and C. Stein, Introduction to Algorithms. MIT Press, 1990.

[25] M. R. G.B. Dantzig, W. Blattner, "Finding a cycle in a graph with minimum cost to time ratio with applications to a ship routing problem," in Theory of graphs, P.Rosenthiel, Ed. Dunod, 1966, pp. 77-83.

[26] M. Gondran and M. Minoux, Graphs and algorithms, 1st ed. John Wiley and sons, 1984.

[27] A. Dasdan, S. Irani, and R. K. Gupta, "Efficient algorithms for optimum cycle mean and optimum cost to time ratio problems," in Design Automation Conference, 1999, pp. 37-42. [Online]. Available: citeseer.ist.psu.edu/dasdan99efficient.html 